\title{
Correlative Light and Electron Microscopy Techniques: Challenges and Successes.
}

Erin S. Stempinski ${ }^{1}$, Xufeng $\mathrm{Wu}^{2}$, and Christine A. Brantner ${ }^{1,3}$

${ }^{1 .}$ Electron Microscopy Core Facility, National Heart Lung and Blood Institute, National Institutes of Health, Bethesda, Maryland, USA.

2. Light Microscopy Core Facility, National Heart Lung and Blood Institute, National Institutes of Health, Bethesda, Maryland, USA.

3. Center for Microscopy and Image Analysis, The George Washington University, Washington, D.C., USA.

Correlative light and electron microscopy (CLEM) combines the strengths of light microscopy (LM) and electron microscopy (EM) to form a more complete picture of a cellular process. LM allows for a multitude of fluorescent tags and a wide field of view to select rare events, and EM allows for increased resolution and visualization of cellular ultrastructure [1]. We used three methods to examine external and internal morphologies of cells: cell cultures grown on coverslips, resin sections on coverslips, and resin sections on coated slot grids. Coverslip methods used indium-tin-oxide (ITO) coverslips with fiducial markers. The coverslips were coated with a $0.1 \%(\mathrm{w} / \mathrm{v})$ Poly-L-lysine solution for $30 \mathrm{~min}$, rinsed in water, and dried on filter paper overnight. Cells were grown and labeled on the coverslips depending on the requirements of the projects and imaged in a laser scanning confocal microscope (LSCM). All samples were imaged on a ZEISS Sigma HD VP Scanning Electron Microscope (SEM). Areas of interest on coverslip samples noted in LM were found in the SEM using ZEISS Shuttle and Find software. Slot grids were placed in a STEM holder and imaged using a backscatter detector. Correlation and overlay of images was performed using multiple software packages.

Cell cultures on ITO coverslips were fixed, dehydrated, and either critical-point dried or treated with hexamethyldisilazane (HMDS). Fine cellular processes of N2A cells were broken or lost in the critical point dryer (CPD), but retained when the cells were en bloc stained with uranyl acetate and dried with HMDS. Challenges of working with the ITO coverslips included fragility, expense, and the requirement of a special holder to be made for the CPD.

Some samples were embedded in LR White resin and serial sections were placed on ITO coverslips with fiducial markers using uncoated slot grids. The coverslip was immunolabeled for LM, imaged with a LSCM, post-stained using $1 \%$ uranyl acetate and lead citrate, then imaged in the SEM. Sections that were $200 \mathrm{~nm}$ in thickness had more fluorescent staining in the LSCM than $100 \mathrm{~nm}$ sections. However, the $200 \mathrm{~nm}$ sections did not provide the necessary resolution in the SEM to image certain ultrastructural features (Figure 1). For both cell culture and resin sections on coverslips, the ZEISS Shuttle and Find software allowed for simple and fast localization of the cell or region of interest between LM and EM.

Samples that used the resin sections on slot grids method were cells cultured on MatTek dishes with gridded coverslips. Cells were fixed, en bloc stained, dehydrated, and infiltrated with Epon resin in the dishes. For some samples, the coverslip was removed from the dish and placed over a round disc mold filled with resin. For other samples, BEEM capsules were placed in the dish. After polymerization, serial sections were cut and placed on coated slot grids. There were challenges releasing the MatTek coverslips from the polymerized resin puck. They did not release when immersed in liquid nitrogen, when heated on a hot plate, nor when separated using a razor blade. Coating the dishes with carbon 
prior to growing the cells did not enhance the separation of the coverslip from the resin. Some glass did release from the resin puck when the coverslip was scored with a diamond scribe. These problems were prevented when BEEM capsules were placed over the region of interest. However, the grid pattern on the coverslip could not be seen once the coverslip was in resin. Therefore, the region of interest needed to be marked on the underside of the coverslip with a diamond scribe prior to processing. DIC images of the region of interest prior to EM processing facilitated both close trimming of the block face near the region of interest and the ability to find the particular cells of interest. The SEM provided a wide field of view, facilitating the ability to find the cells of interest within the section.

Imaging parameters at the LM level determined the success of the LM-EM overlays. Successful overlays had LM images at magnifications similar to those taken at the EM level and LM image resolutions (i.e. pixel numbers) similar to EM image resolutions (Figure 2).

Correlative microscopy is a powerful tool that can be used in the understanding of biological processes. It necessitates robust techniques and attention to detail to ensure success. These techniques can be flexibly adapted for many types of samples based on the information desired [2].

\section{References:}

[1] AA Mironov and GV Beznoussenko, Journal of Microscopy 235 (2009), p.308-321.

[2] Special thanks to the Drs. Panpan Yu, Xueting (Tina) Jin, Lois Greene, and Chad Donaldson for providing project ideas and cell cultures. This research was supported by the Intramural Research Program of the NIH, NHLBI.
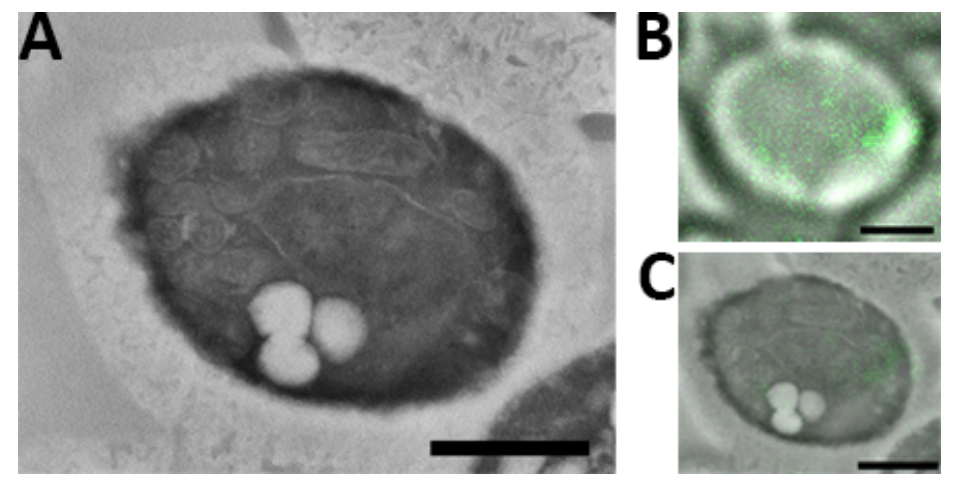

Figure 1. Correlation of $200 \mathrm{~nm}$ sections on ITO coverslips with (A) SEM, (B) LSCM, and (C) overlay of SEM and LSCM. Fine ultrastructural detail in the brightly staining region in (B) is not evident in the SEM. Bar = $1 \mu \mathrm{m}$.

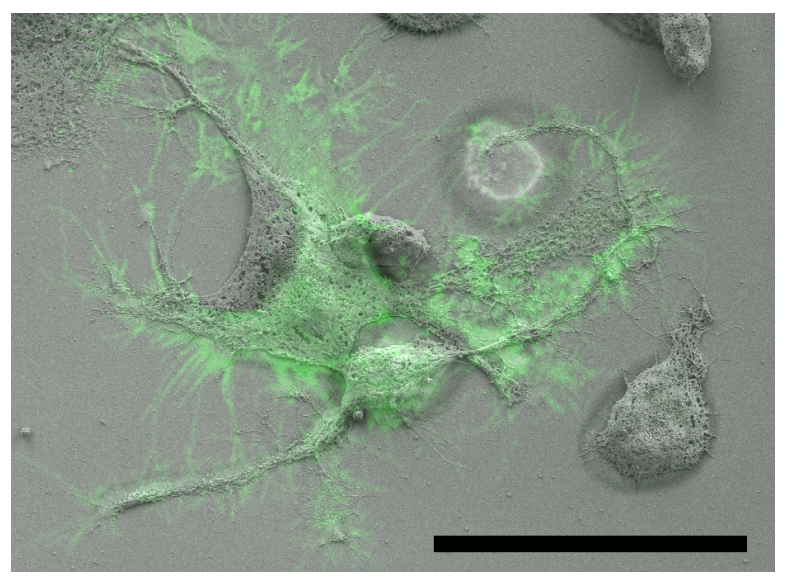

Figure 2. Overlay of LM and EM of N2A cells. Cells were imaged live, fixed, cytoskeleton extracted, and processed for EM. Some shifting occurred due to the cells moving prior to fixation and during processing. Bar $=$ $50 \mu \mathrm{m}$. 